Bulletin of Mathematical Biology (2000) 62, 429-450

doi:10.1006/bulm.1999.0160

Available online at http://www.idealibrary.com on IDE $\mathbf{A}$.

\title{
Wavelengths of Gyrotactic Plumes in Bioconvection
}

\author{
S. GHORAI AND N. A. HILL ${ }^{\dagger}$ \\ Department of Applied Mathematics, \\ University of Leeds, \\ Leeds LS2 9JT, U.K. \\ E-mail: N.A.Hill@leeds.ac.uk
}

\begin{abstract}
Bioconvection occurs as the result of the collective behaviour of many microorganisms swimming in a fluid and is realized as patterns similar to those of thermal convection which occur when a layer of fluid is heated from below. We consider the phenomenon of pattern formation due to gyrotaxis, an orientation mechanism which results from the balance of gravitational and viscous torques acting on bottom-heavy micro-organisms. The continuum model of Pedley et al. (1988, J. Fluid. Mech. 195, 223-237) is used to describe the suspension. The system is governed by the Navier-Stokes equations for an incompressible fluid coupled with a micro-organism conservation equation. These equations are solved numerically using a conservative finite-difference scheme. To examine the dependence of the horizontal pattern wavelengths on the parameters, we consider two-dimensional solutions in a wide chamber using rigid side walls. The wavelengths of the numerical computations are in good agreement with the experimental observations and we provide the first computational examples of the commonly seen 'bottom-standing' plumes.
\end{abstract}

(C) 2000 Society for Mathematical Biology

\section{INTRODUCTION}

Bioconvection is the term used to describe the phenomenon of spontaneous pattern formation in suspensions of micro-organisms such as bacteria and algae (Pedley and Kessler, 1992). In all cases, the micro-organisms are 3\% to 5\% denser than water and on average they swim upwards (although the reasons for up-swimming may be different for different species). Micro-organisms respond to certain stimuli by swimming, on average, in particular directions. These responses are called taxes, examples being gravitaxis, phototaxis, chemotaxis and gyrotaxis. Gravitaxis indicates swimming opposite to gravity, phototaxis denotes swimming towards or away from light and chemotaxis corresponds to swimming up chemical gradients. Gyrotaxis is swimming directed by the balance of torques due to gravity acting on a bottom-heavy cell and shear flow.

Gravitaxis in small micro-organisms is a passive orientation mechanism unlike active responses to external stimuli such as chemotaxis or phototaxis. In large cells

\footnotetext{
${ }^{\dagger}$ Author to whom correspondence should be addressed.
}

$0092-8240 / 00 / 030429+22 \quad \$ 35.00 / 0$

(c) 2000 Society for Mathematical Biology 
or in multicellular organisms, the force of gravity can be 'sensed' dynamically by cilia or statically by movable organelles such as statoliths and otoliths. However, such mechanisms are not usually found in the smaller single cells, which are often simply orientated by their own asymmetry. Cells swim randomly but, for example, if a neutrally buoyant cell is bottom heavy (i.e. its centre of gravity is posterior to its centre of buoyancy), the cell will tend to swim vertically upwards in the absence of any other stimuli resulting in (negative) gravitaxis (Kessler, 1985a). Such cells are also gyrotactic in that a local velocity gradient will produce viscous torques on the cell's body tending to tip it away from the vertical. If the cells do tend to swim upwards, the top layer of the suspension becomes denser than the layer below. When the governing parameters are above critical values, this leads to convective instability and formation of convection patterns. This phenomenon is known as 'bioconvection'; it has some similarity with Rayleigh-Bénard convection, but is driven solely by the swimming of micro-organisms as the following observations make clear. Direct thermal convection can occur in micro-organism suspensions if the containing chamber is heated from below or from the sides, or if sufficient heat is absorbed from the illumination. However, bioconvection continues in a layer that is strongly cooled from below, so it is not a thermal effect (Platt, 1961). The radius, $a$, of a typical gyrotactic cell is approximately $5 \times 10^{-4} \mathrm{~cm}$ and the specific gravity is approximately 0.05 . The Stokes velocity, which is $2 a^{2} \Delta \rho / 9 \mu \approx 3 \times 10^{-4} \mathrm{~cm}$ $\mathrm{s}^{-1}$, is $1 \%$ of the cells' swimming speed and the patterns disappear when the cells stop swimming.

Plesset and Winet (1974) made some measurements of the wavelengths of the bioconvection patterns at the onset of instability in a suspension of the ciliate, Tetrahymena pyriformis, which is negatively gravitactic (but not apparently gyrotactic) (Kessler, 1985a) and showed agreement with the linear stability theory for a layer of dense fluid overlying a layer of light fluid. Kessler (1985a) demonstrated that many swimming micro-organisms are gyrotactic and made observations (Kessler, 1985b) of both the almost regular patterns that occur in concentrated algal suspensions in shallow layers a few millimetres deep, and of gyrotactic plume formation in a tall narrow cylindrical tube. (Childress et al., 1975) analysed the bioconvective instability of a suspension of gravitactic cells, and Pedley et al. (1988) extended the theory of Childress et al. (1975) to develop a continuum model for a suspension of gyrotactic micro-organisms. Figure 2(d) in the review by Pedley and Kessler (1992) shows bioconvection plumes in a tall vessel but no quantitative measurements of such plumes in deep chambers have yet been made. The fluid speed generated within bioconvection is typically of the order of $1 \mathrm{~mm} \mathrm{~s}^{-1}$.

Observations of pattern formation have been recorded previously by such authors as Wager (1911), Loeffer and Mefferd (1952), Wille and Ehret (1968) and Kessler (1984) but the results have tended to be of a qualitative nature. A recent quantitative study of bioconvection in algal suspensions was conducted by Bees and Hill (1997), who measured the wavelengths of the planforms of shallow bioconvection patterns as a function of the depth and concentration of the suspension. Hill 


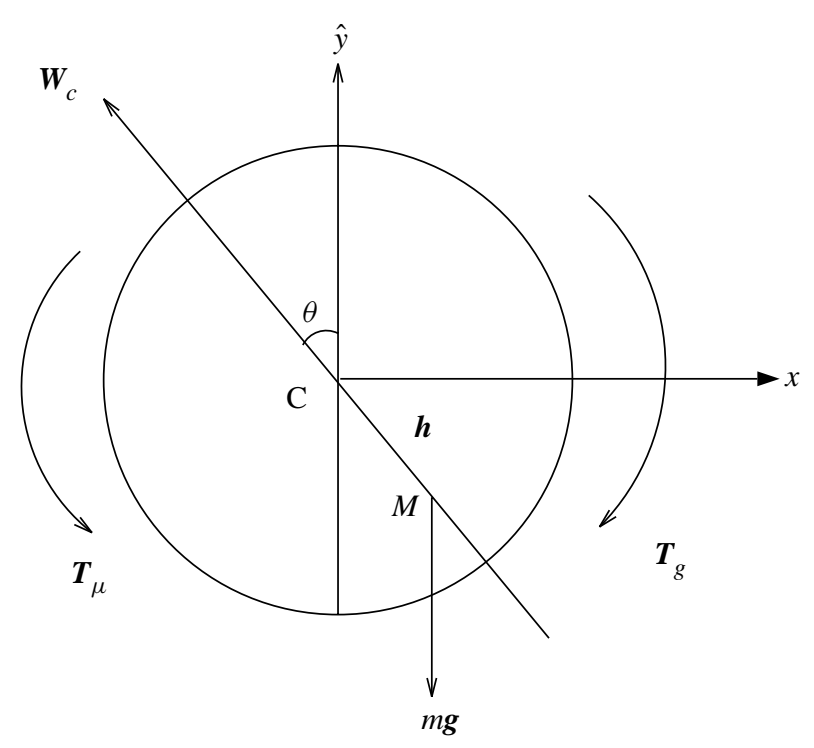

Figure 1. An idealized algal cell. $\theta$ increases in the anti-clockwise sense and $\boldsymbol{h}$ denotes the displacement of the centre of gravity from the centre of the cell, $\mathbf{C}$, so that $\boldsymbol{h}=h(\hat{\boldsymbol{x}} \sin \theta-$ $\hat{\boldsymbol{y}} \cos \theta)$ relative to Cartesian coordinates, $\mathrm{C} x y$. The horizontal, $x$, and the vertical, $y$, directions are fixed relative to the laboratory. $\boldsymbol{W}_{c}$ is the swimming velocity of the microorganisms relative to the water.

and Häder (1997) measured the trajectories of individual swimming algal cells and showed that their motion is well-characterized by the limit of a correlated, biased, random walk in which the time step tends to zero. They were able to calculate the statistical moments required for the coefficients of the Fokker-Planck equation for the cells' orientational probability density function. These coefficients are needed in the new continuum model developed by Pedley and Kessler (1990). Numerical simulation of bioconvection in two dimensions has been carried out by Childress and Peyret (1976) and by Harashima et al. (1988). Both studies were for purely up-swimming (negatively gravitactic) micro-organisms.

For simplicity, algal cells such as Chlamydomonas (whose shapes closely approximate a spheroid) are idealized here as spheres of radius $a$. Figure 1 shows such a cell placed in a shear flow. Since algal cells are small with typical body diameters of $10-20 \mu \mathrm{m}$, and swim at speeds of $100 \mu \mathrm{m} \mathrm{s}^{-1}$, the Reynolds number associated with swimming is very small and inertia can be neglected. Thus a typical cell swims in a direction $\boldsymbol{p}$ at an angle $\theta$ to the vertical determined by a balance between the gravitational torque, $\boldsymbol{T}_{g}$, due to its being bottom heavy, and a viscous torque, $\boldsymbol{T}_{\mu}$, due to fluid-velocity gradients, $\nabla \boldsymbol{u}$, across its body and rotation of the cell, i.e.

$$
\boldsymbol{T}_{g}+\boldsymbol{T}_{\mu}=0
$$

This balance is known as gyrotaxis (Kessler, 1985b). For a sphere of radius $a$, 
the viscous torque is

$$
\boldsymbol{T}_{\mu}=4 \pi \mu a^{3}(\nabla \times \boldsymbol{u}-2 \boldsymbol{\Omega}),
$$

where $\boldsymbol{\Omega}$ is the cell's angular velocity and $\mu$ is the viscosity of the fluid. The rate-of-strain tensor gives rise to an additional torque on aspherical bodies only (Batchelor, 1970). Also, the gravitational torque can be written as

$$
\boldsymbol{T}_{g}=-h m \boldsymbol{p} \times \boldsymbol{g},
$$

where $m$ is the mass of the cell and $g$ is the acceleration due to gravity. For algal cells, $h$ is a few per cent of the cell radius. Simplifying equation (1), we obtain

$$
\frac{\mathrm{d} \theta}{\mathrm{d} t}=\frac{B \zeta-\sin \theta}{2 B}
$$

where $\zeta$ is the horizontal component of the vorticity. $B=4 \pi \mu a^{3} / m g h$ is the time scale for the reorientation of the micro-organisms by the gravitational torque against viscous resistance, and it is called the 'gyrotactic orientation parameter' by Pedley and Kessler (1987).

Thus gravity and vorticity can orient individual cells and guide their trajectories. Gyrotaxis can be demonstrated in an experiment in a slow Poiseuille flow down a vertical tube of circular cross-section. The balance between gravitational and viscous torques gives one stable equilibrium orientation with individual cells tipped away from the upward vertical towards the axis of the pipe. The cells swim towards the axis as they are carried along in the pipe flow, and focus into a narrow beam. Conversely, if the direction of the flow is reversed, the cells are oriented away from the axis toward the walls, confirming the role of gyrotaxis in cell orientation. Gravity also enters in another, entirely different manner in producing co-operative phenomena (Kessler, 1985b). The local average fluid density in a suspension is modified by the presence of cells. If there is a small region with a greater than average concentration of cells, the excess density is sufficient to produce a substantial sinking velocity. The sinking region produces a fluid velocity field which guides further gyrotactic accumulation perpendicular to it. This positive feedback generates and maintains the sharply focused descending plumes of cells that are frequently observed in dense algal cultures. The focused beam of algae is often observed to develop an instability in the form of regularly-spaced axisymmetric 'blobs' (Kessler, 1985b). The blobs are regions of increased cell concentration, which are wider than the beam. They fall faster than the centreline velocity and therefore have an internal vortex-ring structure.

Ghorai and Hill $(1999,2000)$ examined the structure and stability of a single plume in a chamber with either stress-free side walls or periodic side walls. The horizontal wavelength of the plume was specified in advance. The plume exhibited varicose and meandering instabilities, which were explained using a linear stability analysis for an infinitely long plume. Here we choose a wide chamber so that 
there is more than one plume in the chamber. We examine the spacings between the plumes and their dependence on the parameters of the problem. The mathematical formulation of the continuum model is outlined in Section 2. In Section 3, a brief description of the computational method is given, and the results of the numerical computations are presented in Section 4. Finally, in Section 5, we make comparisons with the experimental observations and draw conclusions.

\section{Mathematical Formulation}

The geometry considered consists of a two-dimensional rectangular box of finite width and finite height referred to Cartesian coordinates with the $y$-axis pointing upwards. The top wall of the chamber is open to the air and the fluid satisfies a stress-free boundary condition. The bottom and the side walls are rigid.

2.1. Governing equations. As in Pedley et al. (1988), we assume a monodisperse cell population which can be modelled by a continuous distribution. The suspension is dilute so that the volume fraction of the cells is small and cell-cell interactions are negligible. Each cell has a volume $\vartheta$ and density $\rho+\Delta \rho$, where $\rho$ is the density of the water in which the cells swim and $\Delta \rho / \rho \ll 1$. The velocity $\boldsymbol{u}$ is solenoidal and the vorticity, $\boldsymbol{\omega}=\operatorname{curl} \boldsymbol{u}=(0,0, \zeta)$; thus we introduce a stream function $\psi$ such that

$$
\boldsymbol{u}=(u, v, 0)=\left(\frac{\partial \psi}{\partial y},-\frac{\partial \psi}{\partial x}, 0\right), \quad \zeta=-\nabla^{2} \psi
$$

Conservation of cells requires that the number of cells per unit volume, $n$, satisfies the equation

$$
\frac{\partial n}{\partial t}=-\nabla \cdot J
$$

where the flux of cells is

$$
\boldsymbol{J}=n \boldsymbol{u}+n W_{c} \overline{\boldsymbol{p}}-D \nabla n .
$$

The third term on the right-hand side of equation (5) represents the random component of cell locomotion. We assume that the diffusion coefficient $D$ is homogeneous, isotropic and independent of the other parameters of the problem. The second term in equation (5) arises due to the swimming of the cells: $W_{c} \overline{\boldsymbol{p}}$ is the average swimming velocity relative to the fluid and $W_{c}$ is assumed to be constant. $\overline{\boldsymbol{p}}(\boldsymbol{x}, t)$ represents the average orientation of the cells and is estimated from the torque balance equation. The assumptions of constant isotropic $D$ and deterministic $\overline{\boldsymbol{p}}$ in equation (5) are ad hoc and modifications have been considered by Pedley and Kessler (1990) and by Bees et al. (1998). We retain the simpler form for $\boldsymbol{J}$ in 
Table 1. Estimates of typical parameters for a suspension of C. nivalis.

\begin{tabular}{lll}
\hline Mean concentration & $\bar{n}$ & $10^{6}$ cells cm \\
Specific gravity & $\Delta \rho / \rho$ & 0.05 \\
Average radius & $a$ & $5 \times 10^{-4} \mathrm{~cm}$ \\
Centre of gravity offset & $h$ & $10^{-5} \mathrm{~cm}$ \\
Volume per cell & $\vartheta$ & $5 \times 10^{-10} \mathrm{~cm}^{3}$ \\
Swimming speed & $W_{c}$ & $10^{-2} \mathrm{~cm} \mathrm{~s}^{-1}$ \\
Diffusivity of cells & $D$ & $2.5 \times 10^{-4} \mathrm{~cm}^{2} \mathrm{~s}^{-1}$ \\
Kinematic viscosity & $v$ & $10^{-2} \mathrm{~cm}^{2} \mathrm{~s}^{-1}$ \\
Gyrotactic reorientation parameter & $B$ & $3.4 \mathrm{~s}$ \\
\hline
\end{tabular}

equation (5) because it contains the essential features that we wish to model and because improvements appear to lead to quantitative adjustments rather than qualitative changes (Ghorai, 1997). Typical values for these parameters are given in Table 1, which is based on estimates given by Kessler (1986) for a suspension of Chlamydomonas nivalis.

The vorticity evolves according to the equation

$$
\frac{\partial \zeta}{\partial t}+\nabla \cdot(\zeta \boldsymbol{u})=v \nabla^{2} \zeta-\frac{\Delta \rho g \vartheta}{\rho} \frac{\partial n}{\partial x} .
$$

Here $v$ is the kinematic viscosity and equation (6) is derived under the Boussinesq approximation, neglecting all effects of the cells on the fluid except their negative buoyancy, because the suspension is dilute.

From Fig. 1, we have

$$
\boldsymbol{p} \equiv\left(p_{x}, p_{y}\right)=(-\sin \theta, \cos \theta),
$$

where $\theta$ is the solution of equation (2). If the shear is sufficiently small so that $|B \zeta| \leq 1$, then the steady-state orientation is obtained by setting the left-hand side of equation (2) equal to zero. When $|B \zeta| \leq 1$, we find that

$$
\overline{\boldsymbol{p}}=\left(-\kappa,\left(1-\kappa^{2}\right)^{1 / 2}\right), \quad|\kappa| \leq 1,
$$

where $\kappa=B \zeta$. If the vorticity is large $(|B \zeta|>1)$, the cell tumbles but swims on average in a fixed direction at an angle to the vertical (Kessler, 1985b). When the vorticity is large, the average swimming direction $\overline{\boldsymbol{p}}$ is approximated by integrating the swimming direction over the tumbling period (Ghorai and Hill, 1999).

$$
\text { If } \kappa=B \zeta>1 \text {, then } \quad \bar{p}=\left(-\kappa+\left(\kappa^{2}-1\right)^{1 / 2}, 0\right)
$$

and similarly, if $\kappa=B \zeta<-1$, then

$$
\overline{\boldsymbol{p}}=\left(-\kappa-\left(\kappa^{2}-1\right)^{1 / 2}, 0\right) .
$$


Equations (7), (8) and (9) determine the average swimming direction of the cells for any value of $\zeta$.

The equations are made dimensionless by scaling all lengths on $H$, the depth of the chamber, time on the diffusive time scale $H^{2} / D$, and the bulk fluid velocity on $D / H$, where $D$ is the constant diffusivity of the cells. The cell concentration $n$ is scaled on $\bar{n}$, the mean concentration. The resulting system of coupled equations is

$$
\begin{gathered}
\boldsymbol{u}=(u, v, 0)=\left(\frac{\partial \psi}{\partial y},-\frac{\partial \psi}{\partial x}, 0\right), \quad \zeta=-\nabla^{2} \psi, \\
\frac{\partial \zeta}{\partial t}+\nabla \cdot(\zeta \boldsymbol{u})=S_{c} \nabla^{2} \zeta-S_{c} R \frac{\partial n}{\partial x},
\end{gathered}
$$

and

$$
\frac{\partial n}{\partial t}=-\nabla \cdot J
$$

where the flux of cells is

$$
\boldsymbol{J}=n \boldsymbol{u}+n V_{c} \overline{\boldsymbol{p}}-\nabla n .
$$

(For convenience, we use the same notation for the dimensional and dimensionless variables.) Here, $S_{c}=v / D$ is the Schmidt number, $V_{c}=W_{c} H / D$ is the scaled cell swimming speed and $R$ is a Rayleigh number defined as

$$
R=\frac{\bar{n} \vartheta \Delta \rho g H^{3}}{\rho \nu D}
$$

$\overline{\boldsymbol{p}}$ is defined by equations (7)-(9), where $\kappa=G \zeta$ and $G=B D / H^{2}$ is defined as the gyrotaxis number. The Rayleigh number $R$ is based on the height of the chamber unlike the non-standard $R$ that was used by Ghorai and Hill $(1999,2000)$ to investigate the effects of varying the depth of a fixed-width chamber.

2.2. Initial and boundary conditions. We impose rigid, no-slip boundary conditions at the bottom wall and assume that the top boundary is stress free, so that

$$
\begin{aligned}
u=v=0 & \text { at } y=0, \\
v=\zeta=0 & \text { at } y=1, \\
\boldsymbol{J} \cdot \hat{\boldsymbol{y}}=0 & \text { at } y=0,1,
\end{aligned}
$$

where $\hat{\boldsymbol{y}}$ is the vertical unit vector. The boundary conditions at the rigid side walls are

$$
u=v=\boldsymbol{J} \cdot \hat{\boldsymbol{x}}=0 \quad \text { at } x=0, \lambda,
$$

where $\lambda=$ width $/ H$ is the aspect ratio parameter and $\hat{\boldsymbol{x}}$ is the horizontal unit vector. 
The initial conditions are that of zero flow together with a small perturbation to a uniform concentration of cells. In one case, we consider a random perturbation to a uniform concentration of cells, thus

$$
\psi=0, \quad \zeta=0 \quad \text { and } \quad n=1+\epsilon A(x, y),
$$

where $\epsilon=10^{-5}$ and $A(x, y)$ is a random number between $-\frac{1}{2}$ and $\frac{1}{2}$ at $(x, y)$. We also consider sinusoidal perturbations to a uniform concentration of cells of the following form:

$$
\psi=0, \quad \zeta=0 \quad \text { and } \quad n=1+\epsilon \cos \left(\frac{\nu_{0} \pi x}{\lambda}\right)
$$

where $\epsilon= \pm 10^{-5}$ and $v_{0}$ is an integer. $v_{0}$ together with the sign of $\epsilon$ imposes a specific number of plumes in the initial conditions.

\section{Numerical Procedure}

The governing equations (10)-(12) are discretized using a conservative finitedifference scheme (Ghorai, 1997) on a staggered mesh with the stream function and vorticity stored on one set of nodes and the concentration stored on another set of nodes. The grid is chosen so that the concentration nodes lie in the interior only, whereas those of the stream function and vorticity lie in the interior and also on the boundary of the domain. The advantage of the staggered mesh is that the no-cell flux boundary condition can be satisfied immediately when discretized, without further approximation. We know that there are boundary layers at the top and bottom walls due to the large cell concentration and the presence of the rigid wall, respectively. In order to resolve these gradients accurately, a non-uniform coordinate mesh is used. We transform the non-uniform spatial increments $\Delta x_{i}$ and $\Delta y_{j}$ in the finite-difference equations to a uniform grid using an orthogonal transformation $\xi_{k}=\xi_{k}\left(x_{k}\right)$ which also maps the problem to the computational domain $-1 \leq \xi_{k} \leq 1$, where $x_{k}=(x, y)$ and $\xi_{k}=(\xi, \eta)$. An accurate transformed finite-difference equation for the first-order derivative is given by

$$
\frac{f_{i+1}-f_{i-1}}{2 \Delta \xi(\mathrm{d} x / \mathrm{d} \xi)_{i}}=f_{i}^{I}\left[1+\frac{(\Delta \xi)^{2}}{6}\left(\frac{\mathrm{d}^{3} x}{\mathrm{~d} \xi^{3}}\right)_{i} /\left(\frac{\mathrm{d} x}{\mathrm{~d} \xi}\right)_{i}\right]
$$

(de Rivas, 1972). Here, $f_{i}$ is the value of the function $f(x)$ at the $i$ th node and $f_{i}^{I}$ is the first-order derivative at the $i$ th node. The second term inside the bracket on the right-hand side of equation (20) is important only when there is a large grid variation. The second-order difference operator is obtained by recursion of equation (20). The above difference approximation has a truncation error of $O\left(\Delta \xi^{2}\right)$ 
for an arbitrary mesh transformation in problems of boundary layer character. The stretching transformation is applied in the vertical direction only. Here we have taken the transformations proposed by Roberts (1970).

An expression for the vorticity boundary condition can be obtained by expanding the stream function near the rigid surface using a three-term Taylor series expansion and by making use of the no-slip condition:

$$
\zeta_{\mathrm{w}}=-\frac{\zeta_{\mathrm{nw}}}{2}-3.0 \frac{\psi_{\mathrm{nw}}}{(\Delta n)^{2}}
$$

where $\zeta_{\mathrm{nw}}, \psi_{\mathrm{nw}}$ are the values of $\psi, \zeta$ at the near-wall node (adjacent to the wall) and $\Delta n$ is the non-dimensional distance of the near-wall node from the wall.

An implicit scheme with Euler backward differencing in time and central differencing in space is used to obtain the transient solutions. A line-by-line tridiagonal matrix algorithm with relaxation is used to solve the nonlinear discretized equations. To validate the code, written in terms of stretched coordinates, the code has been run for the heat convection problem and the agreement with the benchmark solution of De Vahl Davis (1983) (Ghorai, 1997) is good. The code has also been used to compute the critical Rayleigh numbers against wavelengths (Ghorai and Hill, 1999) and the agreement with the linear stability results of Hill et al. (1989) is excellent.

\section{Results}

We examine the effects of depth and cell concentration on the wavelengths of the pattern. To this end, we take a wide chamber of $5.0 \mathrm{~cm}$ width to minimize the effect of the side walls on the wavelengths of the patterns. Values of parameters are calculated on the basis of the physical quantities given in Table 1. Using these parameters, we compute solutions for different values of the concentration and depth of the suspension. The dimensional depth and the concentration values are chosen from the experimental data of Bees and Hill (1997). We compute the fully developed pattern wavelengths and compare them with their experimental estimates.

4.1. Evolution of the bioconvection patterns. We consider the first set of experimental data from Bees and Hill (1997) for depth $H=0.333 \mathrm{~cm}$ and background concentration $\bar{n}=2.75 \times 10^{6}$ cells cm $\mathrm{cm}^{-3}$. The evolution of the cell concentration contours starting from a random perturbation is shown in Fig. 2. The concentration of cells at the top of the chamber increases with time due to the cells swimming upwards and accumulating at the top, where the no-cell flux condition holds. The key parameter describing the pattern wavelength is the number of plumes in the box, but care has to be taken with plumes that are adjacent to the walls. At around $t=40 \mathrm{~s}$, plumes begin to develop at the top of the chamber. The number of plumes 
at $t=1 \mathrm{~min}$ is 14 and becomes 15 at $t=1.2 \mathrm{~min}$ with two plumes very close to each other. These two plumes merge to a single plume at around $t=1.7 \mathrm{~min}$ but two of the plumes are located near the two side walls. Thus the mean wavelength at $t=1.7 \mathrm{~min}$ is $5 / 13 \approx 0.385 \mathrm{~cm}$. At $t=8.0 \mathrm{~min}$, the number of plumes remains at 14 but all of them are located in the interior of the chamber and thus the mean wavelength becomes $5 / 15 \approx 0.333 \mathrm{~cm}$. The solution almost reaches the final state, which is steady, as can be seen from the solution at $t=16 \mathrm{~min}$. This solution is referred to as the $28^{+}$solution. The classifications used are that $2 m^{+}$means $m$ plumes in the interior and none along the wall, $2 m^{-}$indicates $(m-1)$ plumes in the interior and two plumes along the walls, whereas $(2 m+1)^{+(-)}$signifies $m$ plumes in the interior with one plume along the right (left) wall (Lennie et al., 1988).

The above solution is not unique. As an example in the next run, we start with a sinusoidal perturbation [see equation (19)] with $v_{0}=16$. The number of plumes in the steady state is 9 with none lying along the wall (see Fig. 3). The wavelength of the plumes is $5 / 10 \approx 0.5 \mathrm{~cm}$. This solution is the $18^{+}$solution.

Harashima et al. (1988) proposed minimum potential energy as a principle for the determination of the steady-state roll size in a suspension of purely up-swimming cells for a given value of Rayleigh number and a given box size. We have plotted the evolution of the kinetic energy against potential energy in Fig. 4. It is clear that the final solution with random perturbation as the initial condition has a higher potential energy than the solution with sinusoidal perturbation $\left(v_{0}=16\right)$ as the initial condition. There exist several other solutions of different wavelengths that can be found by starting with different values of $v_{0}$ as the initial condition. When the value of $v_{0}$ (i.e. the number of sinusoidal modes) is increased, the wavelength of the pattern approaches that of the random perturbation. The random perturbation most closely models the initial conditions in real experiments, and so the wavelength of the final state arising from the random perturbation is most likely to be realized in Bees and Hill's (1997) experiments. Thus pattern wavelengths for the gyrotactic suspensions are clearly not selected by the minimum potential energy principle.

Next we consider evolution of plumes in a relatively deep chamber. We choose Bees and Hill's (1997) sixth set of experimental data for a depth $H=0.729 \mathrm{~cm}$ and a background concentration $\bar{n}=1.02 \times 10^{6}$ cells cm$^{-3}$. The evolution of the concentration starting from a random perturbation of cells is plotted in Fig. 5. The number of distinct plumes at $t=2 \mathrm{~min}$ is 11 and thus the approximate wavelength is $5 / 12 \approx 0.417 \mathrm{~cm}$. At $t=2.4 \mathrm{~min}$, the number of plumes remains at 11 and some of them have hit the bottom of the chamber. The number of plumes extending from the top to the bottom of the chamber is only 6 at approximately $t=3.4 \mathrm{~min}$ but there are some incomplete plumes that do not extend to the top. The solution at $t=27.0$ min shows only 7 plumes. The solution is unsteady and the number of plumes varies between 7 and 9. Thus the mean wavelength of the pattern varies between $0.555 \mathrm{~cm}$ to $0.714 \mathrm{~cm}$. The unsteady nature of the solutions can be seen from the phase diagram of the kinetic energy against potential energy (see Fig. 6). If we start with sinusoidal perturbations, the initial patterns depend on the modes 

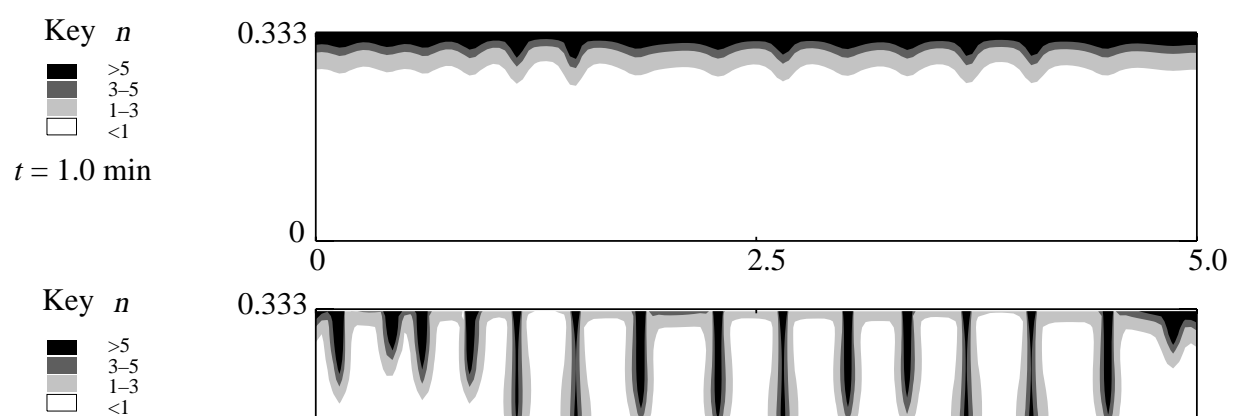

$t=1.2 \mathrm{~min}$
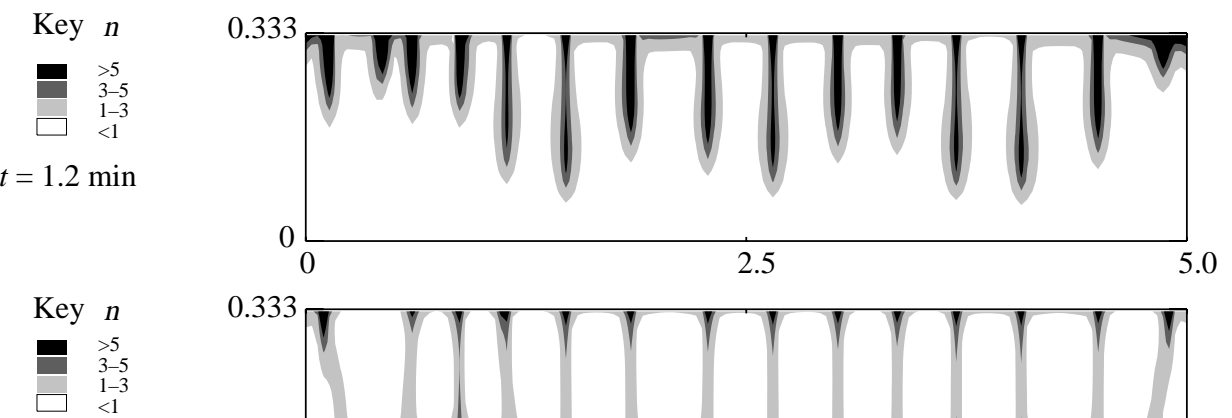

$t=1.7 \mathrm{~min}$
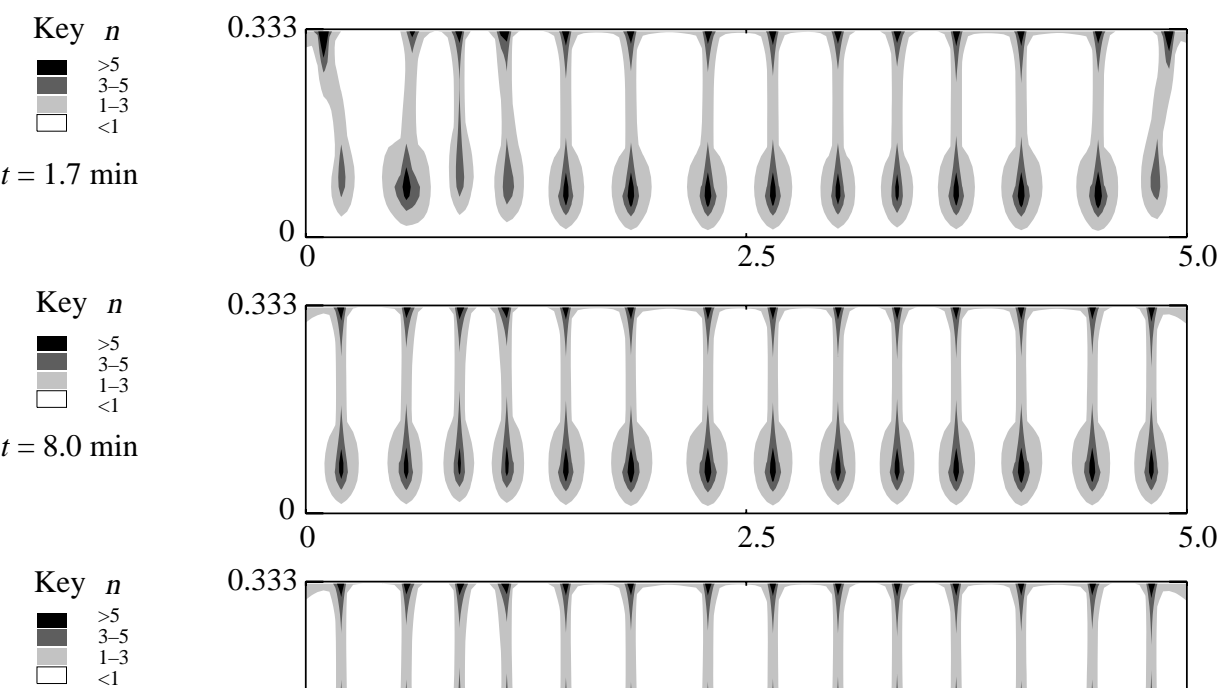

$t=16.0 \mathrm{~min}$

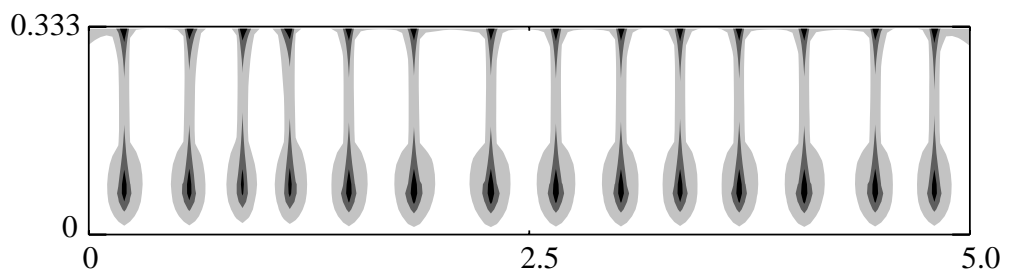

Figure 2. Evolution of the concentration $(n)$ of cells plotted at selected times $(t)$ in a $0.333 \mathrm{~cm}$ deep and $5 \mathrm{~cm}$ wide chamber. The initial conditions consist of no flow and a uniform concentration of cells subject to small random perturbations in concentration. The concentration $n$ is scaled with background concentration $\left(\bar{n}=2.75 \times 10^{6}\right.$ cells cm$\left.{ }^{-3}\right)$. 

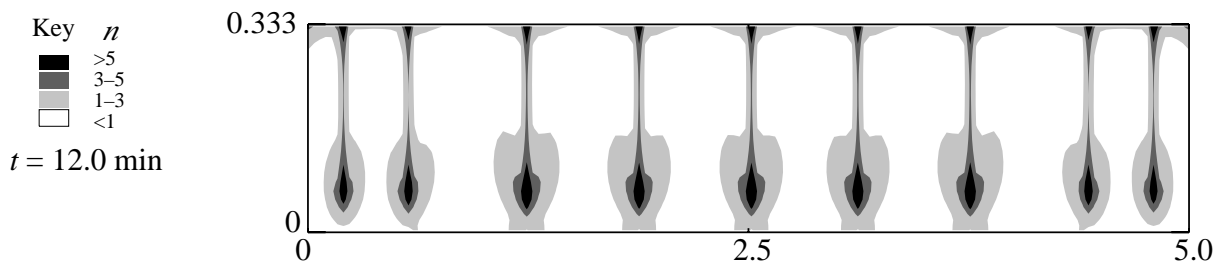

Figure 3. Plume solutions at $t=12 \mathrm{~min}$ in a $0.333 \mathrm{~cm}$ deep and $5 \mathrm{~cm}$ wide chamber. The background concentration is $\bar{n}=2.75 \times 10^{6}$ cells cm$^{-3}$ and the initial conditions consist of no flow and a uniform concentration of cells subject to small sinusoidal perturbations in concentration.

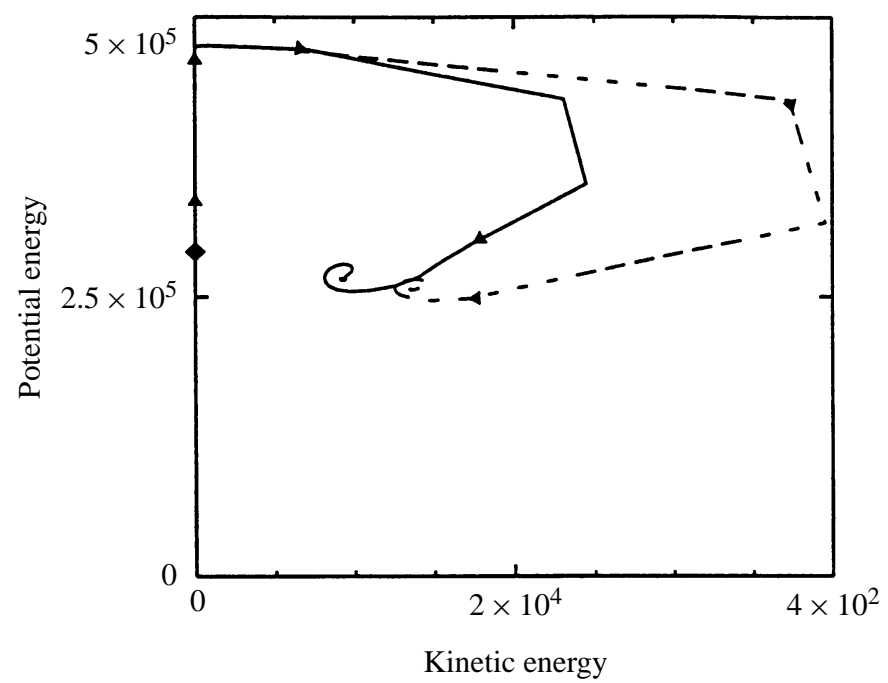

Figure 4. Phase diagram of the dimensionless potential energy vs kinetic energy for the random (solid line) and sinusoidal (broken line) perturbations in concentration. The chamber is $0.333 \mathrm{~cm}$ deep and $5.0 \mathrm{~cm}$ wide with a background concentration of $2.75 \times 10^{6}$ cells $\mathrm{cm}^{-3}$.

of the perturbation but ultimately it approaches an unsteady state similar to that just described. The energy phase diagram also approaches the phase diagram of the random perturbation.

4.2. Effect of varying the depth. Here we consider the effect of the depth on the wavelengths of the pattern keeping other parameters fixed. We choose three sets of data given in experiments 15, 17 and 20 from Bees and Hill (1997). The background concentration is kept fixed at $\bar{n}=1.89 \times 10^{6} \mathrm{cells} \mathrm{cm}^{-3}$ and the depths are $0.318 \mathrm{~cm}, 0.469 \mathrm{~cm}$ and $0.723 \mathrm{~cm}$, respectively. The same random perturbation is applied to all three cases.

The solution at $t=15 \mathrm{~min}$ for the three depths is shown in Fig. 7. The solution becomes steady rapidly for the $0.318 \mathrm{~cm}$ deep chamber and that for the $0.469 \mathrm{~cm}$ deep chamber becomes almost steady after $15 \mathrm{~min}$. However, the solution in the 


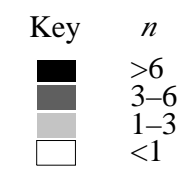

$t=2.0 \mathrm{~min}$
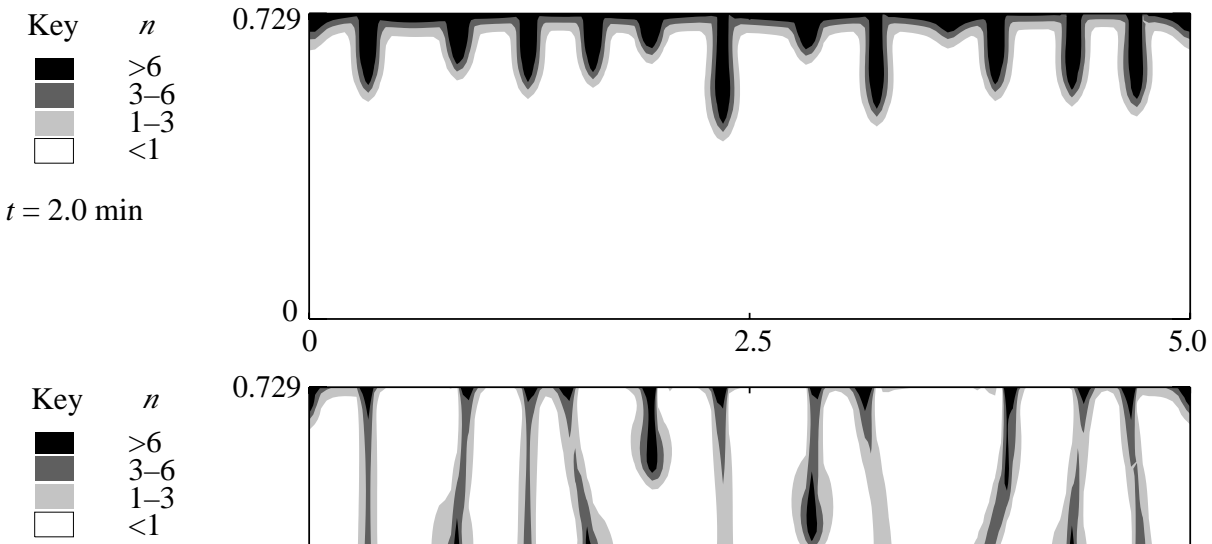

$t=2.4 \mathrm{~min}$
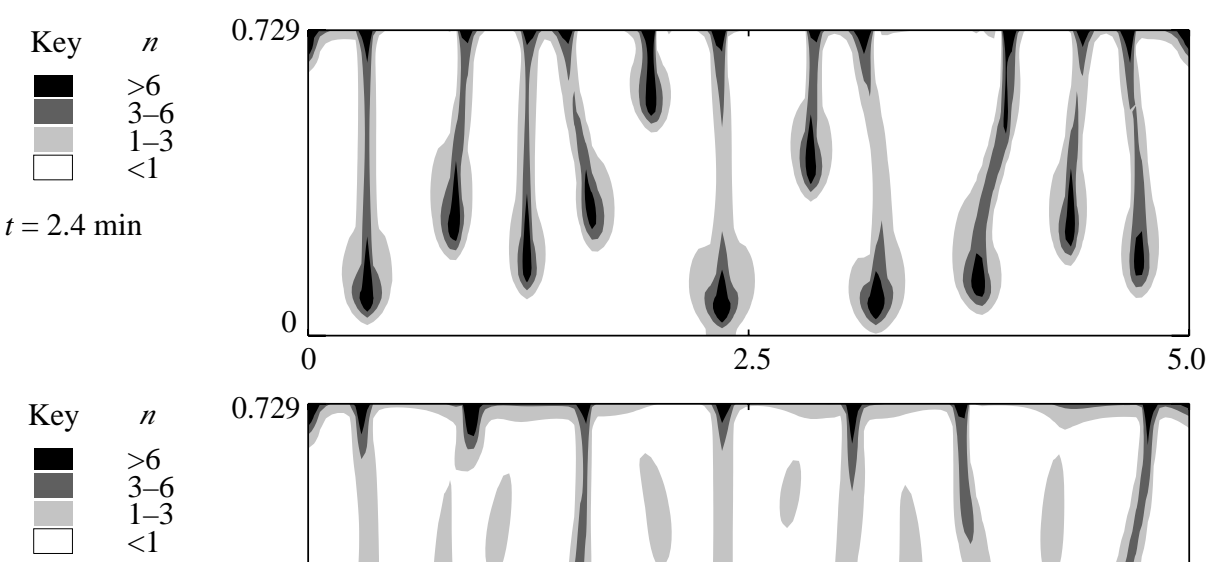

$t=3.4 \mathrm{~min}$
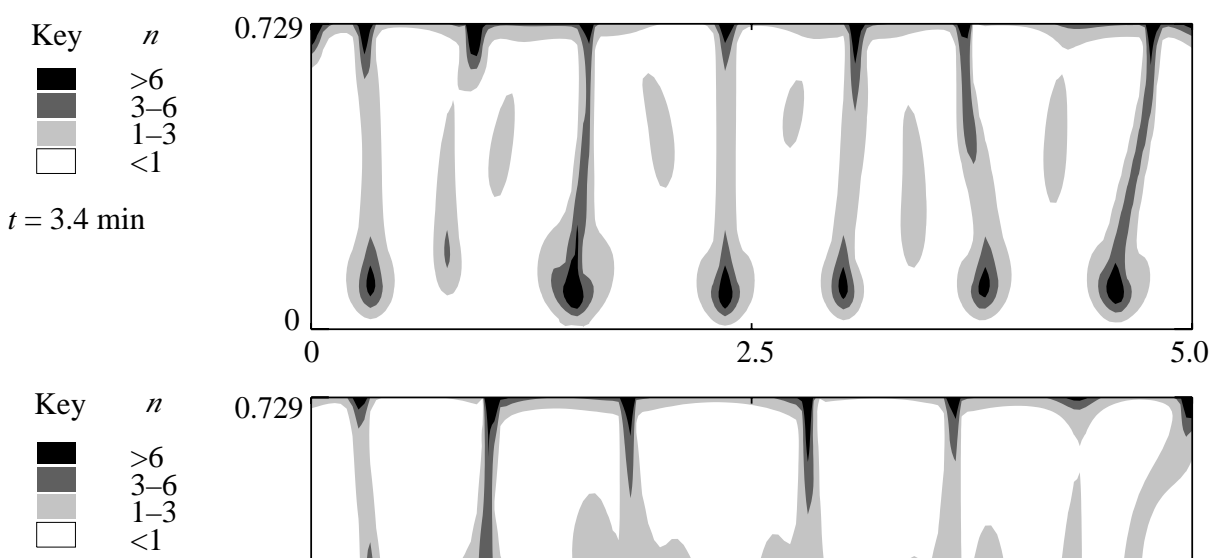

$t=27.0 \mathrm{~min}$

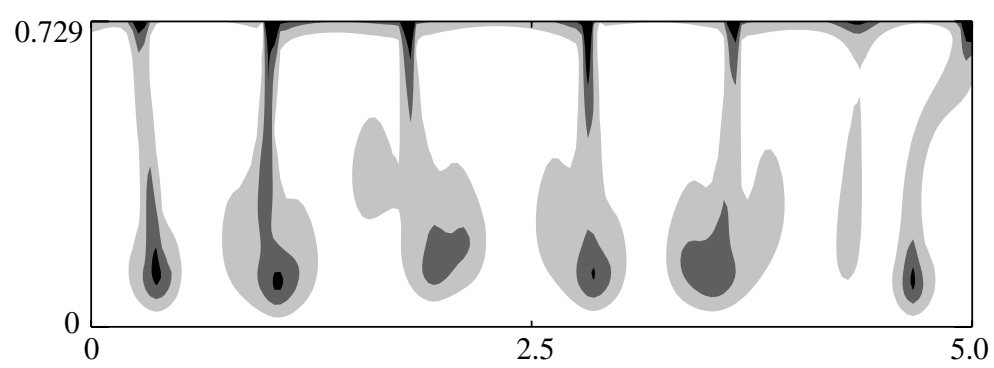

Figure 5. Evolution of the concentration $(n)$ of cells plotted at selected times $(t)$ in a $0.729 \mathrm{~cm}$ deep and $5 \mathrm{~cm}$ wide chamber. The initial conditions consist of no flow and a uniform concentration of cells subject to small random perturbations in concentration. The concentration $n$ is scaled with the background concentration $\left(\bar{n}=1.02 \times 10^{6} \mathrm{cells} \mathrm{cm}^{-3}\right)$. 


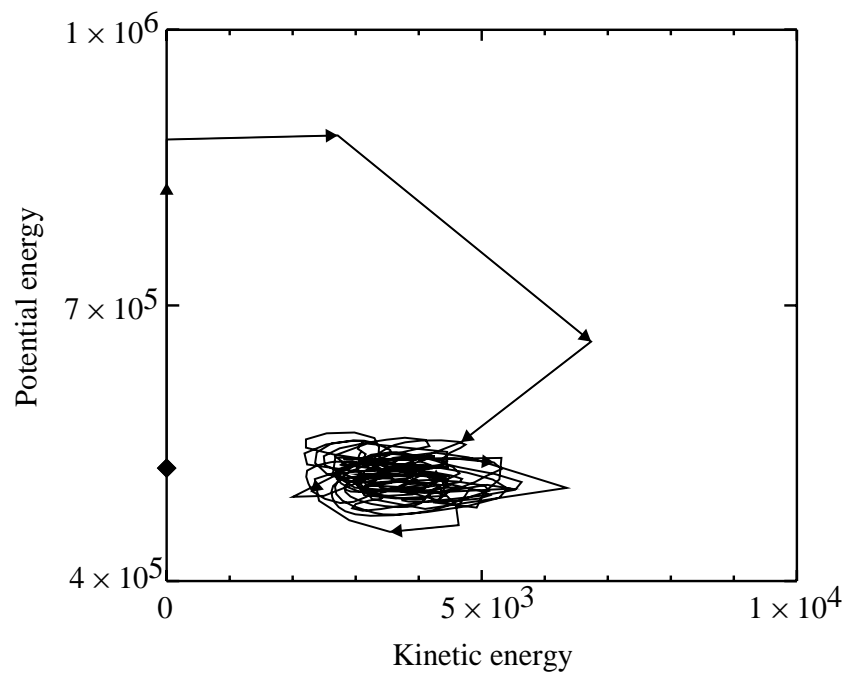

Figure 6. Phase diagram of the dimensionless potential energy vs kinetic energy in a $0.729 \mathrm{~cm}$ deep and $5.0 \mathrm{~cm}$ wide chamber. The background concentration is $1.02 \times 10^{6}$ cells $\mathrm{cm}^{-3}$ and the initial conditions consist of no flow and a uniform concentration of cells subject to small random perturbations in concentration.

$0.723 \mathrm{~cm}$ deep chamber is always unsteady and the number of plumes varies between 11 and 12. Some of the plumes do not extend to the top of the chamber. Similar 'bottom-standing' plumes were observed in experiments (Kessler, 1985b, 1986). The occurrence of bottom-standing plumes transports most of the cells towards the bottom causing wider spacing at the top layer, which was also observed in experiments. In general we find that regularly placed well-defined plumes are not realizable in a deep chamber. The wavelengths of the patterns for the $0.318 \mathrm{~cm}$ and $0.469 \mathrm{~cm}$ deep chambers are approximately $0.357 \mathrm{~cm}$ and $0.417 \mathrm{~cm}$, respectively. Although the plumes are not well defined for the $0.723 \mathrm{~cm}$ deep chamber, the wavelength is still calculated by dividing the total width $(5 \mathrm{~cm})$ by the number of plumes (12) in the chamber. From Fig. 7, we see that the wavelength of the plumes is approximately $0.417 \mathrm{~cm}$ for the $0.723 \mathrm{~cm}$ deep chamber. Thus the wavelength of the pattern increases slightly with the depth of the chamber for the given perturbation.

The solution for the $0.318 \mathrm{~cm}$ deep chamber (see Fig. 7) shows that none of the plumes are located along the walls for the given perturbation but it is possible to obtain such solutions from a different set of random perturbations. To minimize the computing time, we performed four sets of numerical simulations with different random perturbations. The range of wavelengths based on these four simulations together with the experimental values of Bees and Hill (1997) are shown in Table 2 . The wavelengths in the $0.723 \mathrm{~cm}$ deep chamber are not constant due to the unsteady nature of the solutions. On average, the wavelengths of the patterns increase slightly with the depth of the chamber in the numerical results, but decrease 


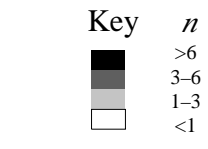

$t=15.0 \mathrm{~min}$

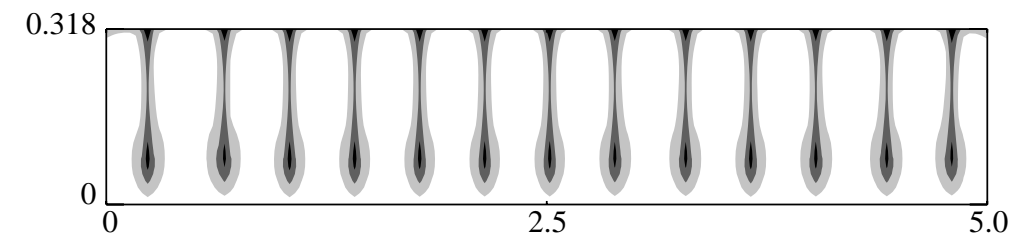

$\begin{array}{cc}\text { Key } & n \\ \square \\ \quad 6 \\ 3-6 \\ 1-3 \\ <1\end{array}$
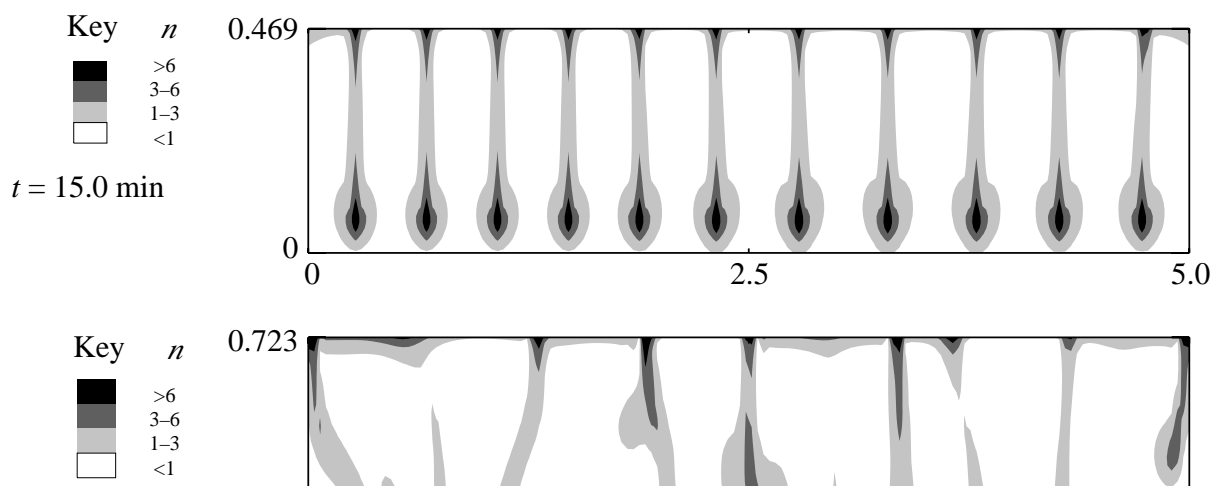

$t=15.0 \mathrm{~min}$

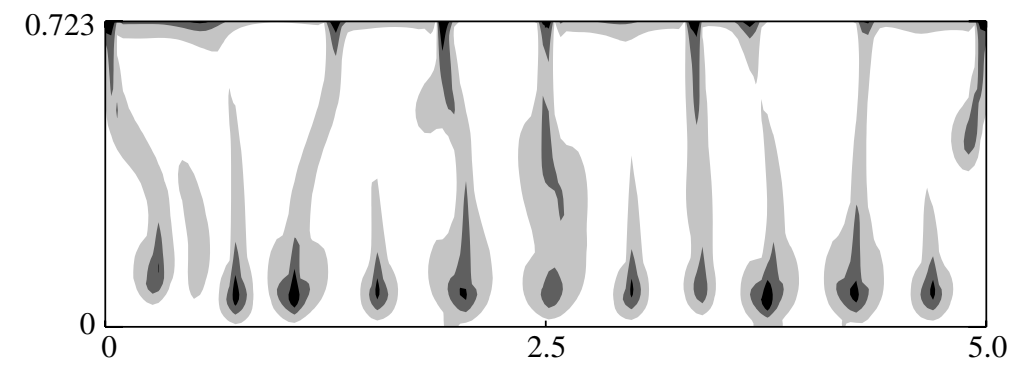

Figure 7. Concentration $(n)$ of cells are plotted at $t=15 \mathrm{~min}$ in chambers of $5 \mathrm{~cm}$ width but of different depths. The initial conditions consist of no flow and a uniform concentration of cells subject to small random perturbations in concentration. The concentration is scaled with the background concentration $\left(\bar{n}=1.89 \times 10^{6}\right.$ cells cm$\left.^{-3}\right)$. 
Table 2. Comparisons between experimental wavelengths and the numerical results. The background concentration is $\bar{n}=1.89 \times 10^{6}$ cells cm$^{-3}$ and the initial conditions consist of no flow and a uniform concentration of cells subject to small random perturbations in concentration. The numbers in the second column refer to the experiment numbers in Bees and Hill (1997).

\begin{tabular}{ccccccc}
\hline \multirow{2}{*}{$\begin{array}{c}\text { Suspension } \\
\text { depth }\end{array}$} & \multirow{2}{*}{$\begin{array}{c}\text { Expt. } \\
\text { no. }\end{array}$} & $\begin{array}{c}\text { Wavelength } \\
(\text { experimental })\end{array}$ & \multicolumn{5}{c}{$\begin{array}{c}\text { Wavelength (numerical) } \\
(\mathrm{cm})\end{array}$} \\
\cline { 5 - 7 } & & $(\mathrm{cm})$ & $(1)$ & $(2)$ & $(3)$ & $(4)$ \\
\hline 0.318 & 15 & 0.489 & 0.357 & 0.385 & 0.333 & 0.357 \\
0.469 & 17 & 0.303 & 0.417 & 0.417 & 0.417 & 0.357 \\
& 18 & 0.352 & & & & \\
& 19 & 0.333 & & & & \\
0.723 & 20 & 0.344 & 0.417 & 0.454 & 0.417 & 0.417 \\
\hline
\end{tabular}

Table 3. Comparisons between experimental wavelengths and the numerical results. The background concentration is $\bar{n}=4.19 \times 10^{6}$ cells cm$^{-3}$ and the initial conditions consist of no flow and a uniform concentration of cells subject to small random perturbations in concentration. The numbers in the left column refer to the experiment numbers in Bees and Hill (1997).

\begin{tabular}{ccccccc}
\hline \multirow{2}{*}{$\begin{array}{c}\text { Expt. } \\
\text { no. }\end{array}$} & $\begin{array}{c}\text { Suspension } \\
\text { depth }\end{array}$ & \multirow{2}{*}{$\begin{array}{c}\text { Wavelength } \\
(\text { experimental })\end{array}$} & \multicolumn{4}{c}{$\begin{array}{c}\text { Wavelength (numerical) } \\
(\mathrm{cm})\end{array}$} \\
\cline { 5 - 7 } & $(\mathrm{cm})$ & $(\mathrm{cm})$ & $(1)$ & $(2)$ & $(3)$ & $(4)$ \\
\hline 25 & 0.186 & 0.297 & 0.294 & 0.278 & 0.294 & 0.278 \\
24 & 0.291 & 0.252 & 0.278 & 0.294 & 0.294 & 0.312 \\
23 & 0.468 & 0.234 & 0.357 & 0.333 & 0.357 & 0.357 \\
\hline
\end{tabular}

slightly in the experimental results. However, the trends are not very marked in the experiments as can be seen from experiments 17,19 and 20 where the wavelengths increased slightly with the depth of the chamber.

The concentration values chosen in the above experiments are moderate. Next we choose three sets of data given in experiments 23, 24 and 25 from Bees and Hill (1997) with a higher background concentration $\bar{n}=4.19 \times 10^{6} \mathrm{cells} \mathrm{cm}^{-3}$ and the depths are $0.186 \mathrm{~cm}, 0.291 \mathrm{~cm}$ and $0.468 \mathrm{~cm}$, respectively. The well-developed concentration patterns are shown in Fig. 8. The wavelengths of the patterns are approximately $0.294 \mathrm{~cm}, 0.278 \mathrm{~cm}$ and $0.357 \mathrm{~cm}$, respectively, for a given set of random perturbation. The range of wavelengths based on four simulations together with the experimental values of Bees and Hill (1997) are shown in Table 3. The wavelength decreases slightly with the depth of the chamber in the experiments. As in the previous case, the wavelengths increase slightly with depth in the numerical results.

4.3. Effect of varying the background concentration. We consider the effect of varying the background cell concentration on the wavelengths of the patterns 

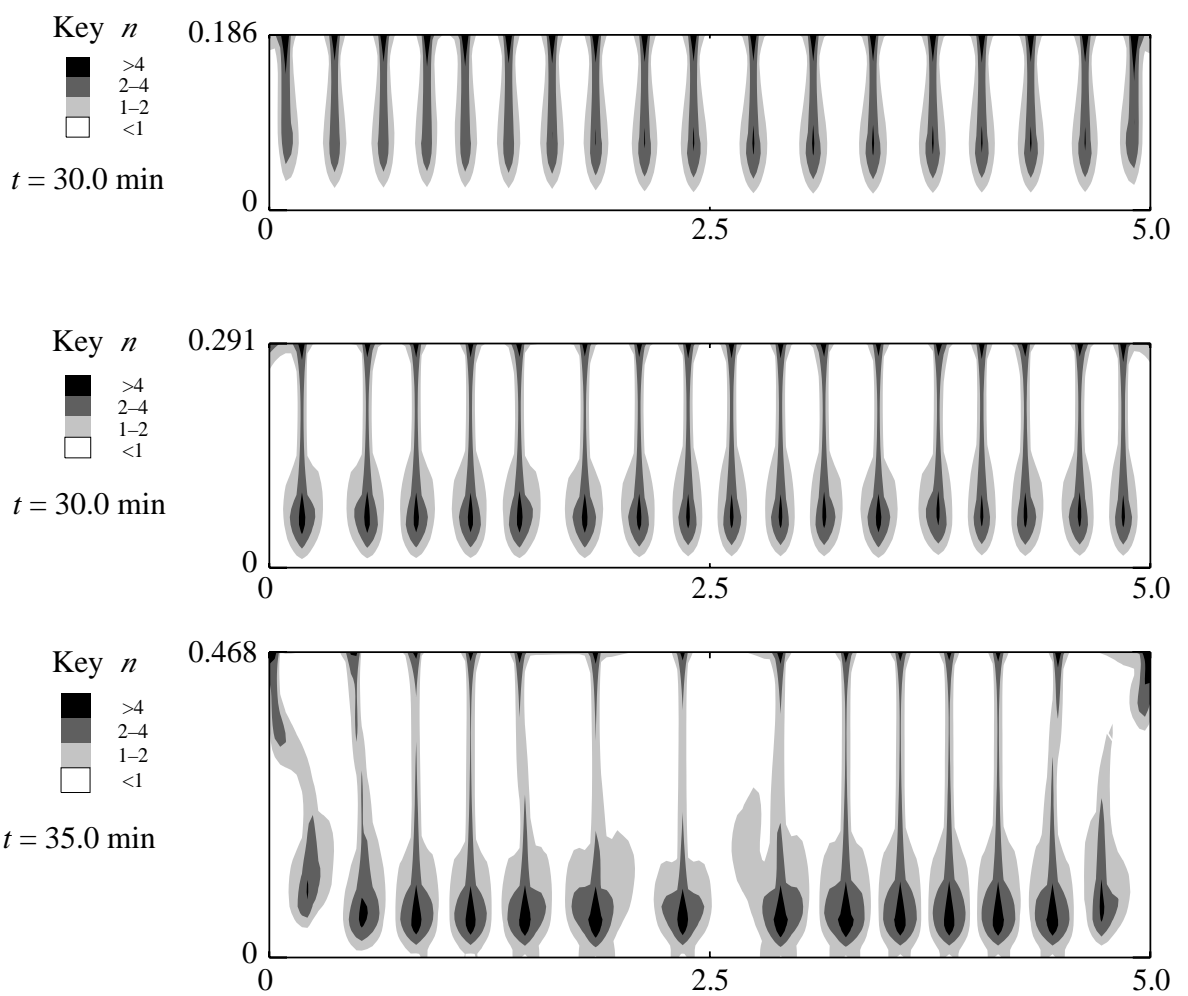

Figure 8. Concentration ( $n$ ) of cells are plotted at selected times in chambers of $5 \mathrm{~cm}$ width but of different depths. The initial conditions consist of no flow and a uniform concentration of cells subject to small random perturbations in concentration. The concentration is scaled with the background concentration $\left(\bar{n}=4.19 \times 10^{6}\right.$ cells cm$\left.^{-3}\right)$. 

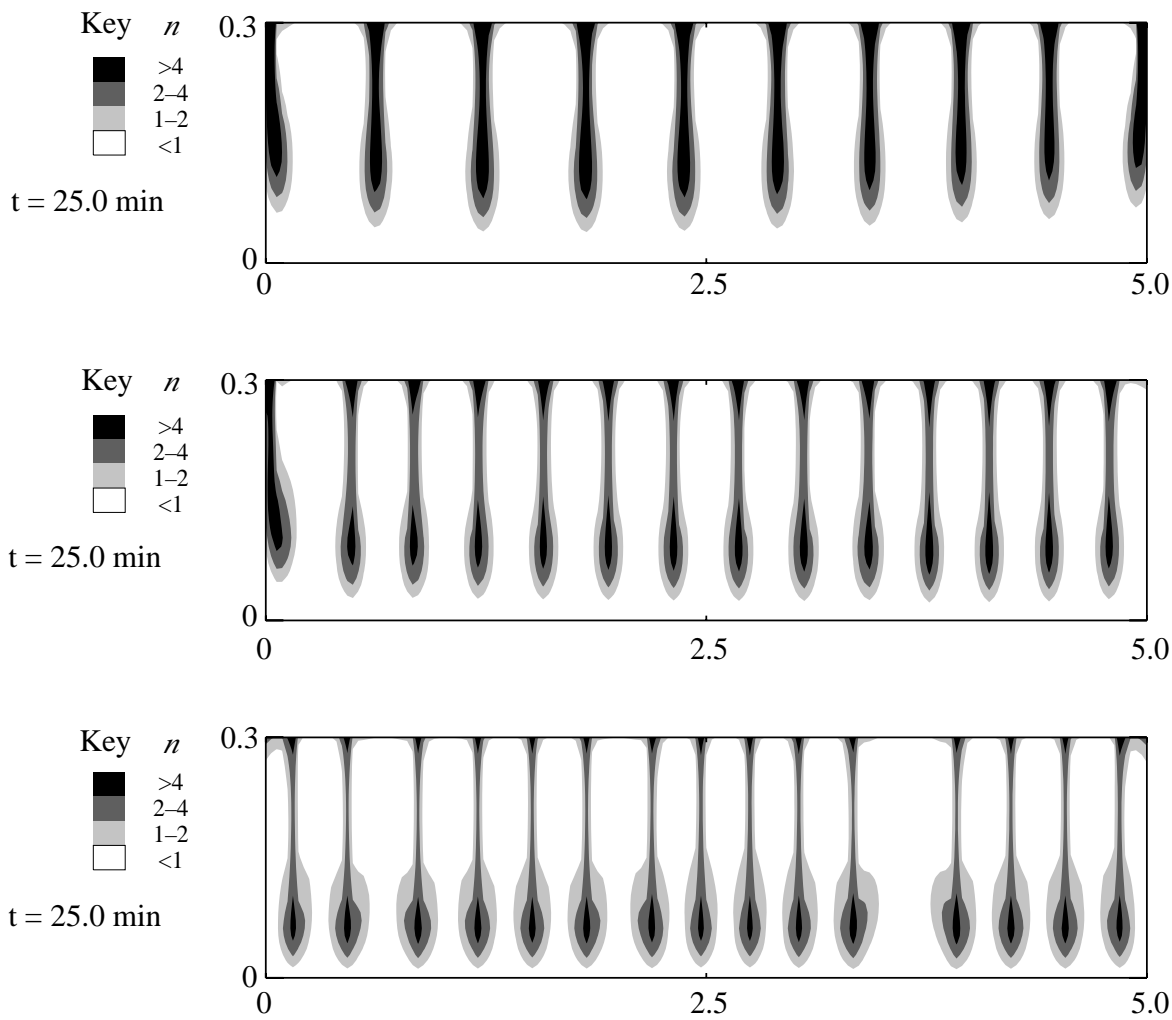

Figure 9. Concentration $(n)$ of cells are plotted at $t=25 \mathrm{~min}$ in a $5.0 \mathrm{~cm}$ wide and $0.3 \mathrm{~cm}$ deep chamber. The background concentrations are $\bar{n}=10^{6}$ cells cm $\mathrm{cm}^{-3}, 2 \times 10^{6}$ cells $\mathrm{cm}^{-3}$ and $4 \times 10^{6}$ cells cm $\mathrm{cm}^{-3}$, respectively. The initial conditions consist of no flow and a uniform concentration of cells subject to small random perturbations in concentration. The concentration is scaled with the background concentration.

whilst keeping other parameters fixed. We use a moderate depth of $0.3 \mathrm{~cm}$ and take three sets of concentration values; $\bar{n}=10^{6}$ cells cm${ }^{-3}, 2 \times 10^{6}$ cells cm$~^{-3}$ and $4 \times 10^{6} \mathrm{cells} \mathrm{cm}^{-3}$, which represent the range covered in the experiments of Bees and Hill (1997).

The solutions for the $0.3 \mathrm{~cm}$ deep chamber are shown in Fig. 9. The numbers of plumes are 10,14 and 15, respectively, and the corresponding wavelengths are approximately $0.5 \mathrm{~cm}, 0.357 \mathrm{~cm}$ and $0.313 \mathrm{~cm}$. The wavelengths of the pattern clearly decrease with an increase in the background concentration of the suspensions, which was also observed by Bees and Hill (1997).

Next we consider solutions in a $0.5 \mathrm{~cm}$ deep chamber with the same background concentrations as in the previous numerical solutions. These are shown in Fig. 10, where the solutions for the $10^{6}$ cells cm$~^{-3}$ and $2 \times 10^{6}$ cells cm$^{-3}$ background concentration values have reached the steady state. The solution for the highest background concentration is unsteady but the plumes are well defined and the number of plumes remains the same at all times. The numbers of plumes are 10, 11 and 

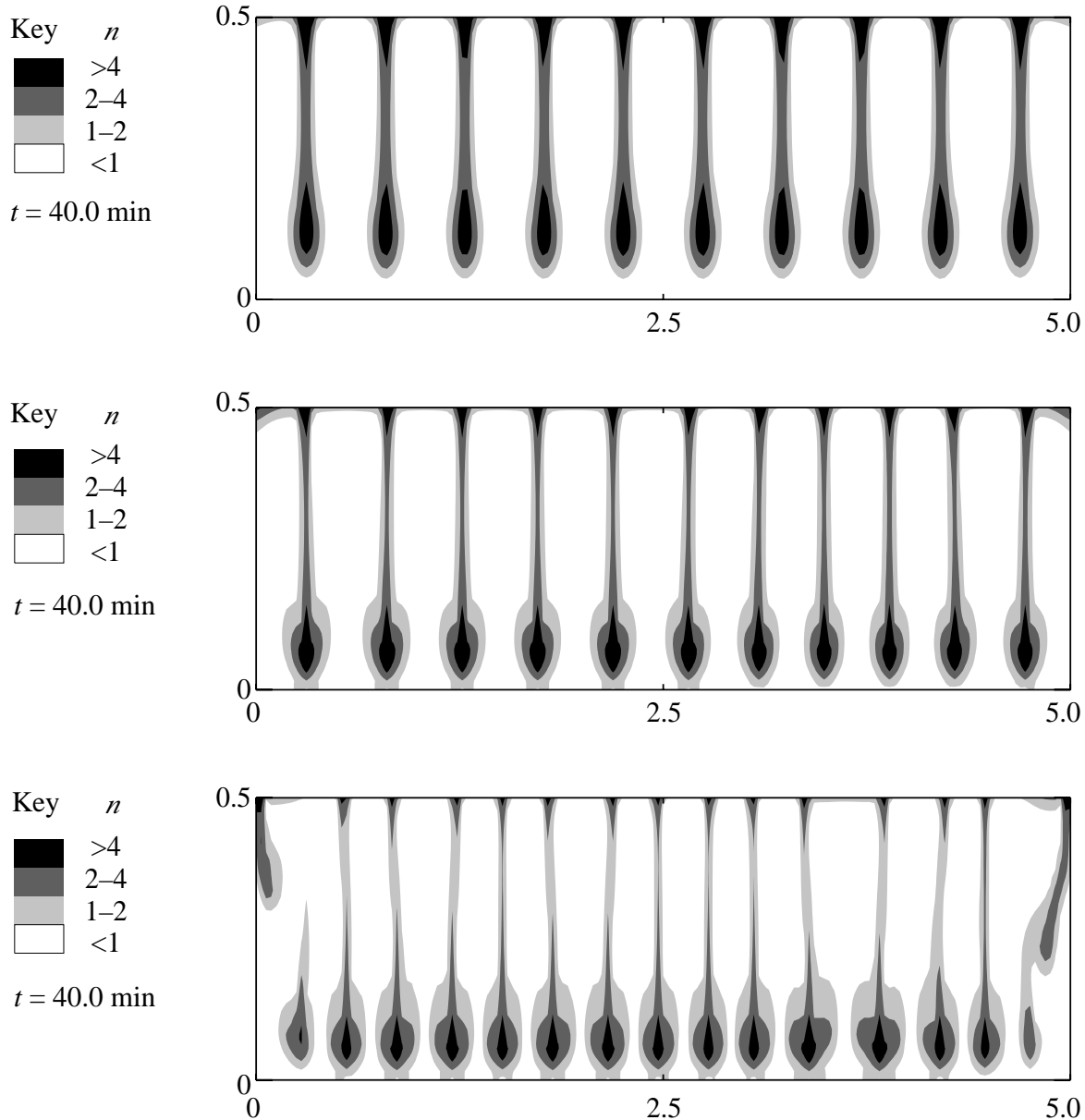

Figure 10. Concentration ( $n$ ) of cells are plotted at $t=40 \min$ in a $5.0 \mathrm{~cm}$ wide and $0.5 \mathrm{~cm}$ deep chamber. The background concentrations are $\bar{n}=10^{6}$ cells cm $\mathrm{cm}^{-3}, 2 \times 10^{6}$ cells $\mathrm{cm}^{-3}$ and $4 \times 10^{6}$ cells $\mathrm{cm}^{-3}$, respectively. The initial conditions consist of no flow and a uniform concentration of cells subject to small random perturbations in concentration. The concentration is scaled with the background concentration. 
15 , respectively, and the corresponding wavelengths are approximately $0.454 \mathrm{~cm}$, $0.416 \mathrm{~cm}$ and $0.357 \mathrm{~cm}$. Again the wavelengths clearly decrease with an increase in the background concentration, in agreement with the experiments.

\section{Conclusions}

We have examined the dependence of the wavelengths on the depth of the chamber and the background concentration of the cells and the following conclusions are based on the numerical experiments reported in the previous sections. The two-dimensional well-developed wavelengths increase slightly with the depth of the chamber according to the numerical results given in Tables 2 and 3. The wavelengths estimated by Bees and Hill (1997) decreased slightly with an increase in the depth of the chamber but the trend is not clearly marked. In contrast, the welldeveloped wavelengths clearly decrease with an increase in the background concentration, in agreement with the experimental results. Bottom-standing plumes have been simulated for the first time. They have two main characteristics. First, they are observed in deep chambers only with a high background concentration of cells and, secondly, bottom-standing plumes are always unsteady. When they occur, most of the cells are transported to the bottom of the chamber and some of the plumes do not extend to the top of the chamber causing wider spacing at the top of the chamber. Previous attempts at modelling bottom-standing plumes (Pedley, 1988) were probably unsuccessful due to the unsteady nature of the plumes.

The differences between the experimental and numerical results might be due to the following reasons. The numerical experiments are two-dimensional whereas the experiments of Bees and Hill (1997) were performed in three dimensions. Also, although we chose a wide chamber to minimize the effect of the side walls, still the side walls select whole numbers of plumes. Thus, a discrete change in wavelength occurs as a plume is added or removed from the pattern. The physical parameters required for the numerical experiments are the best estimates currently available but many are not accurately known. In particular, the solution is most sensitive to the value of the diffusion parameter which is especially hard to estimate. Additionally, our model with isotropic diffusivity and directed swimming is inconsistent, assuming weak randomness to specify $\boldsymbol{W}_{c}$ and strong randomness to specify diffusivity. The new model proposed by Pedley and Kessler (1990) removes these discrepancies and might give better agreement with the experimental results. For simplicity, we have considered spherical cells only, whereas a typical algal cell closely resembles a spheroid. Also, the effects of the flagella are ignored. Despite these discrepancies, the numerical results agree well, both qualitatively and quantitatively, with the experimental observations. 


\section{REFERENCES}

Batchelor, G. K. (1970). The stress system in a suspension of force-free particles. J. Fluid Mech. 41, 545-570.

Bees, M. A. and N. A. Hill (1997). Wavelengths of bioconvection patterns. J. Exp. Biol. 200, 1515-1526.

Bees, M. A., N. A. Hill and T. J. Pedley (1998). Analytical approximations for the orientation distribution of small dipolar particles in steady shear flows. J. Math. Biol. 36, 269-298.

Childress, S., M. Levandowsky and E. A. Spiegel (1975). Pattern formation in a suspension of swimming micro-organisms: equations and stability theory modes. J. Fluid Mech. 69 , 595-613.

Childress, S. and R. Peyret (1976). A numerical study of two-dimensional convection by motile particles. J. Mécanique 15, 753-779.

De Vahl Davis, G. (1983). Natural-convection of air in a square cavity - a bench-mark numerical-solution. Int. J. Numer. Methods Fluids 3, 249-264.

Ghorai, S. (1997). Bioconvection and plumes, PhD thesis, University of Leeds, UK.

Ghorai, S. and N. A. Hill (1999). Development and stability of gyrotactic plumes in bioconvection. J. Fluid Mech. 400, 1-31.

Ghorai, S. and N. A. Hill (2000). Periodic arrays of gyrotactic plumes in bioconvection. Phys. Fluids 12, 5-22.

Harashima, A., M. Watanabe and I. Fujishiro (1988). Evolution of bioconvection patterns in a culture of motile flagellates. Phys. Fluids. 31, 764-775.

Hill, N. A. and D.-P. Häder (1997). A biased random walk model for the trajectories of swimming micro-organisms. J.Theor. Biol. 186, 503-526.

Hill, N. A., T. J. Pedley and J. O. Kessler (1989). Growth of bioconvection patterns in a suspension of gyrotactic micro-organisms in a layer of finite depth. J. Fluid Mech. 208, 509-543.

de Rivas, K. (1972). On the use of nonuniform grids in finite-difference equations. J. Comput. Phys. 10, 202-210.

Kessler, J. O. (1984). Gyrotactic buoyant convection and spontaneous pattern formation in algal cell cultures, in Non-Equilibrium Cooperative Phenomena in Physics and Related Fields, M. G. Velarde (Ed.), New York: Plenum Press, pp. 241-248.

Kessler, J. O. (1985a). Hydrodynamic focusing of motile algal cells. Nature 313, 218-220.

Kessler, J. O. (1985b). Co-operative and concentrative phenomena of swimming microorganisms. Contemp. Phys. 26, 147-166.

Kessler, J. O. (1986). Individual and collective fluid dynamics of swimming cells. J. Fluid Mech. 173, 191-205.

Lennie, T. B., D. P. McKenzie, D. R. Moore and N. O. Weiss (1988). The breakdown of steady convection. J. Fluid Mech. 188, 47-85.

Loeffer, J. B. and R. B. Mefferd (1952). Concerning pattern formation by free-swimming microorganisms. Am. Nat. 86, 325-329.

Pedley, T. J. and J. O. Kessler (1987). The orientation of spheroidal micro-organisms swimming in a flow field. Proc. R. Soc. Lond. Ser. B 231, 47-70. 
Pedley, T. J. (1988). Bottom-standing plumes in gyrotactic bioconvection. Bull. Am. Phys. Soc. 33, 2282 (Abstract).

Pedley, T. J. and J. O. Kessler (1990). A new continuum model for suspensions of gyrotactic micro-organisms. J. Fluid Mech. 212, 155-182.

Pedley, T. J. and J. O. Kessler (1992). Hydrodynamic phenomena in suspensions of swimming micro-organisms. Ann. Rev. Fluid Mech. 24, 313-358.

Pedley, T. J., N. A. Hill and J. O. Kessler (1988). The growth of bioconvection patterns in a uniform suspension of gyrotactic micro-organisms. J. Fluid Mech. 195, 223-237.

Platt, J. R. (1961). 'Bioconvection patterns' in cultures of free-swimming organisms. Science 133, 1766-1767.

Plesset, M. S. and H. Winet (1974). Bioconvection patterns in swimming microorganism cultures as an example of Rayleigh-Taylor instability. Nature 248, 441-443.

Roberts, G. O. (1970). Computational meshes for boundary layer problems, Second International Conference on Numerical Methods in Fluid Dynamics, Lecture Notes in Physics, M. Holt (Ed.), 8, New York: Springer, pp. 171-177.

Wager, H. (1911). On the effect of gravity upon the movements and aggregation of Euglena viridis, Ehrb., and other micro-organisms. Phil. Trans. R. Soc. Lond. Ser. B 201, 333390.

Wille, J. J. and C. F. Ehret (1968). Circadian rhythm of pattern formation in population of a free-swimming organism, Tetrahymena. J. Protozool. 15, 789-792. 